\title{
Transaction
}

\section{Specific Attraction at the Carboxyl Terminus of Fatty Acid/Oxidized Aluminum Interface for the Sizing Appearance of Fiber-network Materials}

\author{
Shingo Yokota*, Kana Matsuyama, Hiroki Yamamoto, Takuya Kitaoka, and Hiroyuki Wariishi
}

\author{
Department of Forest and Forest Products Sciences, Graduate School of Bioresource and Bioenvironmental \\ Sciences, Kyushu University, 6-10-1 Hakozaki, Higashi-ku, Fukuoka 812-8581, Japan ( * Present address: \\ Institute for Chemical Research, Kyoto University, Uji, Kyoto 611-0011, Japan)
}

\begin{abstract}
The attractive force acting at a carboxyl group of a fatty acid/oxidized aluminum interface was precisely determined by atomic force microscopy (AFM). The correlation of the attractive force with the sizing behavior of paper sheets sized by a fatty acid-aluminum sulfate (alum) system was investigated. The surfaces of gold-coated AFM probes were modified with alkylthiol terminating methyl or carboxyl groups through self-assembling chemisorption. Pure cellulose film and oxidized aluminum plate were used as model substrates of cellulosic pulp fibers and aluminum components, respectively, in the sized paper sheet. A strong attractive force was detected at a carboxyl-terminated AFM probe/aluminum oxide interface both in air and water, while very weak interaction was found at the other pairs of probe/ substrates. In the fatty acid-alum systems, it was observed that such a combination was indispensable for good sizing appearance of not only organic pulp fiber sheet, but also inorganic glass filter. Consequently, the specific attractive interaction between carboxyl termini of acid sizing agents and oxidized aluminum components in the alum-treated fiber matrix should play a significant role in the water-repellent properties of porous fiber-network materials.
\end{abstract}

(Received 1 June, 2009; Accepted 29 July, 2009)

\section{Introduction}

Paper-based products have occupied much public attention as eco-friendly, convenient, and multi-purpose materials having a wide range of applications [1-3]. A paper sheet fundamentally has a layered, porous micronetwork architecture consisting of hydrophilic cellulosic pulp fibers. That structure results in excellent liquid absorptive properties, especially for water. The wetting characteristics of solid material surfaces are of great importance from a practical viewpoint. In the case of paper materials, adequate water-repellency, the so-called sizing effect, has been required for improvement of printing quality by ink-bleeding control, runability of coating operation, durability of liquid containers, and other surface characteristics [4]. Thus, sizing phenomena and mechanisms are of great interest for fundamental research and for functional design of cellulosic fiberbased materials.

Paper sizing treatments provide a controllable slowdown of water penetration into the porous fiber network of hydrophilic paper sheets. Control is achieved by using small amounts of sizing agents with proper retention aids, through internal additive addition or surface coating systems. In particular, sizing systems with rosin acids and aluminum sulfate (alum) have long been widely applied in the industrial papermaking process, because they are highly effective, have good cost performance, and are easily used. Rosin acids as a sizing agent are a mixture of amphipathic diterpenes comprising hydrophobic rings and a hydrophilic carboxyl group. Alum, a typical retention aid of rosin sizes, plays key roles in size retention and sizing appearance both in internal and surface sizing treatments [4].

Effective rosin-alum sizing systems have attracted much attention as surface modification processes for cellulosic fiber-based materials. For that reason a great deal of effort has been directed toward such unique sizing systems. Those systems have been discussed on the basis of the chemical reaction between rosin acids and aluminum components [5-9], and the size retention [1013] and distribution on paper sheets [14-19]. In our previous report involving emulsion rosin sizing with alum, it was shown that free size acids in their original form directly contributed to effective sizing appearance of paper sheets, while the aluminum salts of size components were either absent or were minor components [7-9]. Elemental analysis revealed that the aluminum species in alum-added paper sheets were present as oxidized compounds [20,21]. Consequently, an 
interfacial phenomenon between the free carboxyl group of sizing components and the oxidized surfaces of aluminum deposits must be of great significance for sizing appearance.

The present study is focused on the attractive force acting at carboxyl moieties/oxidized aluminum interfaces, which was evaluated by atomic force microscopy (AFM) with a force-distance measurement technique. AFM provides not only ultra-fine three-dimensional images of solid surfaces, but also quantitative information on interfacial interactions at femto- to nanoNewton force resolution in various conditions [22]. In this work, the surfaces of AFM probes were modified with each alkylthiol had either a methyl- or carboxyl-terminus at one end. Regenerated cellulose film and oxidized aluminum plate were used as models of cellulosic pulp fibers and alum components, respectively. A fatty acidalum sizing system $[8,9,18,19]$ was adopted as a model rosin-alum system for elucidation of the correlation between nanoscopic interfacial force at the nanoNewton level, and macroscopic sizing behavior of sized paper sheets. In addition, the findings were applied to sizing treatment of other porous materials comprising hydrophilic fibrous components. In this report, an inorganic glass fiber sheet (glass filter) was subjected to the fatty acid-alum sizing system, and its water-repellent properties were investigated.

\section{Experimental}

\subsection{Materials}

A commercial hardwood bleached kraft pulp was beaten to $460 \mathrm{~mL}$ of Canadian Standard Freeness [23] with a Hollander beater [24], for handsheet-making. Cellulose powder (CF1; Whatman International, Ltd.) was used for preparation of cellulose film on a silicon wafer (NIRACO, Co. Ltd.). Polyvinylamine (PVAm; Mw ca. $1 \times 10^{6} \mathrm{~g} / \mathrm{mol}, \mathrm{Mw} / \mathrm{Mn} c a .2 .4$, charge density $3.8 \mathrm{meq} / \mathrm{g}$ ) was prepared by partial hydrolysis of synthesized polyvinylformamide [13]. A fatty acid mixture consisting of stearic and palmitic acids (Wako Pure Chemical Industries, Co. Ltd.) in equal weight (SPA) was used as a model of rosin sizing agent $[8,9,18,19]$. Aluminum plate (NIRACO, Co. Ltd.) was oxidized in an electric furnace at $500^{\circ} \mathrm{C}$ for more than $3 \mathrm{~h}$; Al-O formation was confirmed by X-ray photoelectron spectroscopy (XPS). Aluminum sulfate $\left(\mathrm{Al}_{2}\left(\mathrm{SO}_{4}\right)_{3}\right.$, alum), $N$-methylmorpholine- $N$-oxide (NMMO), and other chemicals were of pure reagent grade (Aldrich, Co. Ltd.) and used as purchased. The water used in this study was purified with a Milli-Q system (Millipore, Inc.).

\subsection{Sizing treatment}

Alum (0-2.0\% on dry weight of pulp) was added to a $0.15 \%$ pulp suspension ( $\mathrm{pH} 6.5$ ), and handsheets with a basis weight of $60 \mathrm{~g} / \mathrm{m}^{2}$ were prepared [25]. The conditioned handsheets were soaked in a $0.25 \%(\mathrm{w} / \mathrm{v})$ $\mathrm{SPA} /$ chloroform $\left(\mathrm{CHCl}_{3}\right)$ solution for $1 \mathrm{~min}$ and then airdried at $23{ }^{\circ} \mathrm{C}$ and $50 \%$ relative humidity $(\mathrm{RH})$ for at least $24 \mathrm{~h}$ [18]. Glass filter (GA55; Advantec MFS, Inc.) was dipped in $0-0.4 \%$ alum solution and then sodium hydroxide solution was added dropwise to precipitate oxidized aluminum compounds on the surface of the glass fibers. After air-drying, the alum-treated glass filters were subjected to the sizing treatment described above with $0.5 \%(\mathrm{w} / \mathrm{v}) \mathrm{SPA} / \mathrm{CHCl}_{3}$ solution.

\subsection{Cellulose film}

Regenerated cellulose film was prepared according to the previous report [26]. $\mathrm{CF} 1$ powder was dissolved in $80 \%(\mathrm{w} / \mathrm{w}) \mathrm{NMMO} / \mathrm{H}_{2} \mathrm{O}$ solution at $105^{\circ} \mathrm{C}$ for $30 \mathrm{~min}$ : the homogeneous $2.0 \%$ cellulose $/ \mathrm{NMMO} / \mathrm{H}_{2} \mathrm{O}$ solution was then diluted to some extent with dimethylsulfoxide to reduce the viscosity. A silicon wafer was oxidized in an electric furnace at $1000^{\circ} \mathrm{C}$ for $30 \mathrm{~min}$, then soaked in a $0.1 \%$ aqueous solution of PVAm. The polymerpretreated substrate was spin-coated with cellulose solution at $2500 \mathrm{rpm}$ for $10 \mathrm{~s}$. The cellulose film was precipitated with ethanol and then rinsed several times with water, followed by air-drying at room temperature.

\subsection{Force-distance measurement}

A commercially available gold-coated silicon nitride cantilever (NPG; length $c a .115 \mu \mathrm{m}$, spring constant $c a$. $0.58 \mathrm{~N} / \mathrm{m}$, resonance frequency $c a .57 \mathrm{kHz}$, tip radius of curvature 30-60 nm; Veeco Instruments, Inc.) was immersed in $1 \mathrm{mM}$ ethanolic 1-octadecanethiol $\left(\mathrm{CH}_{3}\left(\mathrm{CH}_{2}\right)_{17} \mathrm{SH}, \mathrm{ODT}\right)$ or 11-mercapto-1-undecanoic acid $\left(\mathrm{HOOC}\left(\mathrm{CH}_{2}\right)_{10} \mathrm{SH}, \mathrm{MU}\right)$ solution at room temperature for $48 \mathrm{~h}$ [27]. The modified AFM probes were then washed thoroughly with ethanol, and dried in flowing nitrogen gas. Adhesive force between AFM probes (modified or unmodified) and substrates (cellulose film or oxidized $\mathrm{Al}$ plate) was analyzed in air and pure water by contactmode AFM using a NanoScope IIIa atomic force microscope (Veeco Instruments, Inc.) at $25^{\circ} \mathrm{C}$ and $50 \%$ RH. The maximum load and scan rate were set at $c a$. $20 \mathrm{nN}$ and $2.0 \mu \mathrm{m} / \mathrm{s}$, respectively. Force-distance curves obtained at 30 different points for each sample were analyzed using a Scanning Probe Image Processor (SPIP Version 3.1.0.2, Image Metrology). Adhesive force was calculated according to the following eq. (1): 
Adhesive force $(\mathrm{nN})=$ (Deflection (V)/Cantilever sensitivity $(\mathrm{V} / \mathrm{nm})) \times$ Spring constant $(\mathrm{N} / \mathrm{m})$

\subsection{Size retention analysis}

The total size content of the paper sheets was determined by pyrolysis gas chromatography (PyGC) with on-line methylation using a $25 \%$ tetramethylammonium hydroxide/methanol solution [11]. Surface elemental analysis of the substrates was carried out by the XPS apparatus (AXIS-HSi; Shimadzu/Kratos, Co. Ltd.) equipped with a monochromatic $\mathrm{AlK} \alpha$ X-ray source $(1486.6 \mathrm{eV})$. All XPS measurements were performed using $15 \mathrm{kV}$ voltage and $10 \mathrm{~mA}$ current, and the pressure in the analyzing chamber was maintained below $5 \times 10^{-7}$ $\mathrm{Pa}$. The pass energy and step width for the survey scan were set at $80 \mathrm{eV}$ and $1 \mathrm{eV}$, respectively. For narrow scans those parameters were set at $10 \mathrm{eV}$ and $0.05 \mathrm{eV}$. The binding energies for all spectra were referenced to a $\mathrm{C} 1 \mathrm{~s}$ signal (reduced C-C) at $285.0 \mathrm{eV}$. The atomic ratio of carbon from size to carbon in pulp matrix, indicating the surface abundance of size components present on pulp fibers, was defined by dividing the amounts of unoxidized $(\mathrm{C} 1,285.0 \mathrm{eV})$ and carbonyl $(\mathrm{C} 4,289.3 \mathrm{eV})$ carbons by those of C-O $(\mathrm{C} 2,286.7 \mathrm{eV})$ and $\mathrm{O}-\mathrm{C}-\mathrm{O}(\mathrm{C} 3,288.1 \mathrm{eV})$ originating from cellulose $[18,19]$.

\subsection{Water-repellency test}

The sizing degrees of handsheets were evaluated by conventional Stöckigt sizing test [28]. The contact angle (CA) of water droplets on the glass filter surfaces was measured by the sessile drop technique using a

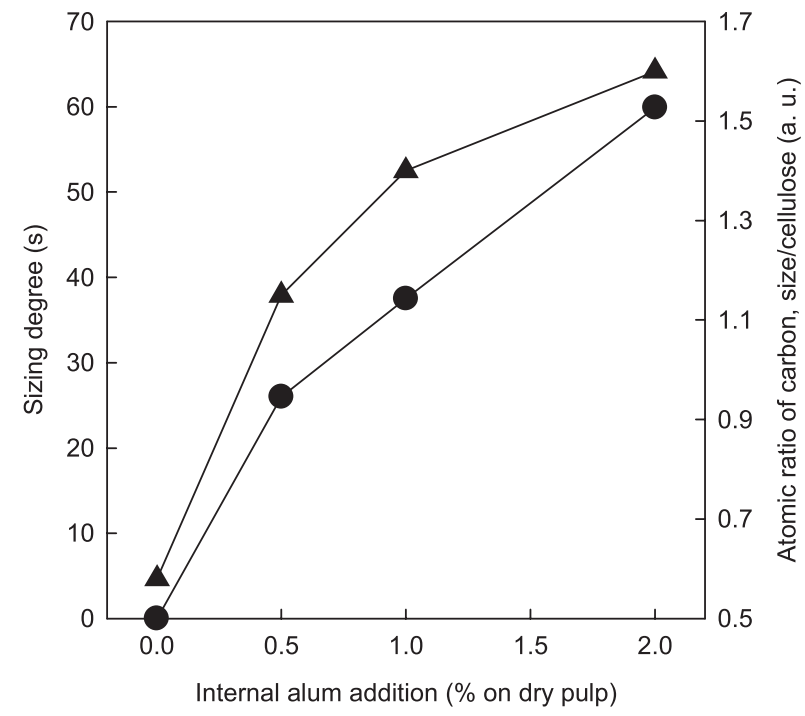

Fig. 1 Sizing degree (circles) and atomic ratio of carbon (triangles) of handsheets sized with $0.25 \% \quad(\mathrm{w} / \mathrm{v}) \quad \mathrm{SPA} / \mathrm{CHCl}_{3} \quad$ solution. The handsheets were pretreated with $0-2.0 \%$ internal alum addition.
DropMaster 500 (Kyowa Interface Science, Co. Ltd.). A water drop $(1.0 \mu \mathrm{L})$ was gently placed on the surface, and its $\mathrm{CA}$ value was determined $\left(25^{\circ} \mathrm{C}, 50 \% \mathrm{RH}\right)$. Images of the water droplets on the substrates were analyzed using the FACE software (ver. 2.0.6) supplied with the apparatus [29]. The CA measurements were repeated at least five times for each sample.

\section{Results and discussion}

\subsection{Sizing behavior of paper sheets sized with fatty acid/chloroform system}

Model sizing of alum-pretreated handsheets with the $\mathrm{SPA} / \mathrm{CHCl}_{3}$ system was carried out, and their fundamental sizing behavior investigated. Figure 1 shows sizing degrees and surface abundance of size components of handsheets soaked in $0.25 \%(\mathrm{w} / \mathrm{v}) \mathrm{SPA} / \mathrm{CHCl}_{3}$ solution. Surface abundances of size components were calculated from the atomic ratios of size components/cellulosic matrix, which was determined by XPS C1s analysis $[18,19]$. Both sizing effects and surface size abundance increased with increase in the internal addition of alum in the handsheet-making process, while no sizing effect appeared in the alum-free paper sheet. Although no electrostatic interaction between size and alum components operates in this nonaqueous system, internal alum addition had a great impact on size retention, resulting in the sizing appearance.

Figure 2 compares the sizing degrees, surface size abundance and total size content, of alum-free and $2 \%$

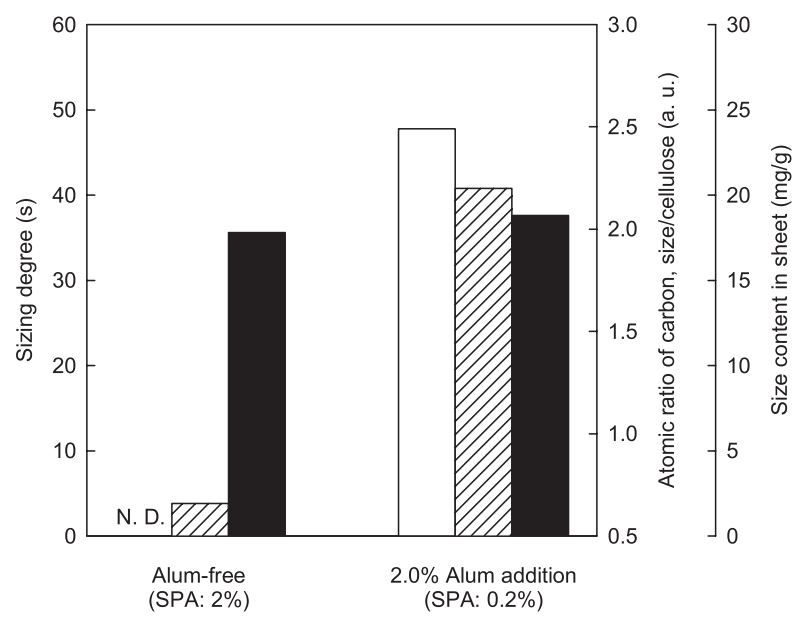

Fig. 2 Sizing degree (open bars), atomic ratio of carbon (striped bars), and total size content (filled bars) of handsheets sized with SPA/ $\mathrm{CHCl}_{3}$ solutions. The concentration of SPA/ $\mathrm{CHCl}_{3}$ solution was adjusted to $2 \%$ (alum-free sheet) and $0.2 \%$ (2.0\% alum-added sheet). 
alum-added paper sheets after sizing treatments. In order to adjust the total size contents in paper sheets which were measured by PyGC, the alum-free and 2\% alumadded handsheets were treated with $2 \%$ and $0.2 \%(\mathrm{w} / \mathrm{v})$ $\mathrm{SPA} / \mathrm{CHCl}_{3}$ solutions, respectively. Extreme differences were found both in the sizing degrees and the surface size abundance, although total size retention was almost the same. A clear sizing effect appeared only in the alumadded paper sheets, while no sizing appearance was detected in the alum-free (fatty acid-treated) sheet. Surface size abundance determined by XPS has been reported to be involved in the size aggregation/ distribution on the sheet surface $[18,19]$. Figure 2 indicates that size components were relatively scattered on the surface of alum-added paper sheets, whereas size components may have formed massive aggregates on the alum-free paper. Thus it was predicted that an essential interaction occurred at an interface of sizing agents and alum components, leading to homogeneous distribution of size components. The sizing system using $\mathrm{SPA} / \mathrm{CHCl}_{3}$ solution is useful for elucidating the rosin-alum sizing mechanism. In order to shed light on such a unique fatty acid-alum interaction in paper sheets, direct detection of interactive forces between size and alum components was investigated by AFM and is discussed in the following sections.

\subsection{Attractive forces acting at surface-modified AFM probes/substrate interfaces}

Figure 3 shows a schematic illustration of surface modification of AFM probes with alkylthiols with different functional termini, namely ODT or MU. The well known spontaneous chemisorption of organosulfur compounds onto a metallic gold surface was utilized to make methyl- or carboxyl-modified AFM probes [27]. In parallel with such simulation of sizing agents, pure cellulose, and oxidized aluminum substrates were adopted as model surfaces of pulp fibers and alum, respectively. Adhesive forces were analyzed by force-distance measurements using such probes and model substrates. An example of the force-distance curve was illustrated in Fig. 4.

Table 1 lists the adhesive forces at each probe/ substrate interface, in air and in deionized water. The much larger forces measured in air than in water. It is known that such larger force in air is attributable to effects of the stronger van der Waals force and the capillary force of water slightly adsorbed on the surfaces of the probes and substrates [22]. The adhesive forces at carboxyl-modified probe/oxidized aluminum were $c a .57$ and $5 \mathrm{nN}$ in air and water, respectively. These values were several times higher than those found at the interfaces of unmodified and methyl-modified probes with cellulose and oxidized aluminum substrates. These results suggested that a unique attractive force was experienced by specific pairs of carboxyl groups and oxidized aluminum surface. The surface density of methyl and carboxyl moieties on the AFM probes is as yet unknown, but the standard deviations were within approximately ten percent, confirming the validity and reproducibility of the measurements. This is the first time that direct detection of interactive force acting at a model interface of size and alum components has been achieved, and may possibly be essential for paper sizing appearance.

The attractive force at the carboxyl group/aluminum oxide interface is presumably allowed to effectively, stably anchor size components on the alum-added paper sheets. Unique interaction between fatty acids and aluminum compounds has been reported in fields other than paper chemistry. For example, aluminum interfaces

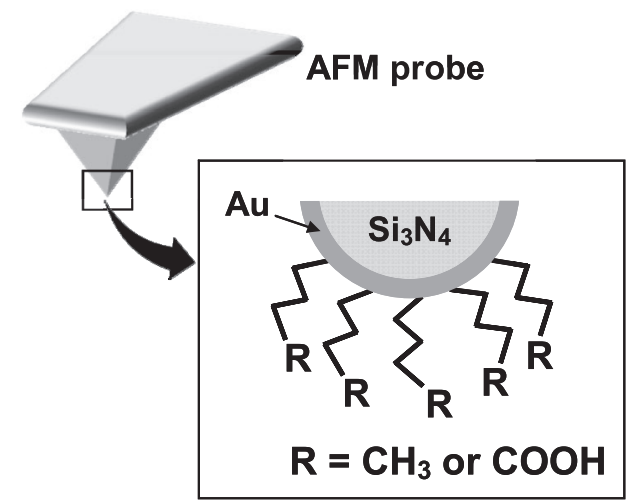

Fig. 3 Schematic illustration of surface modification of AFM probes with alkylthiols having different functional termini. R: $\mathrm{CH}_{3}$, 1-octadecanethiol; $\mathrm{COOH}, 11-$ mercapto-1-undecanoic acid.

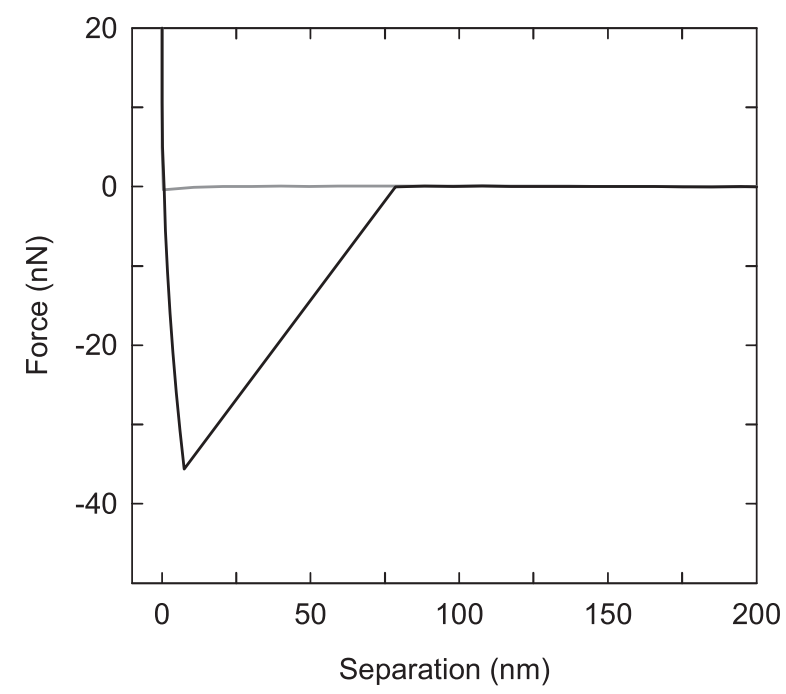

Fig. 4 Force-distance plot between a gold-coated AFM probe and a cellulose substrate. 
Table 1 Attractive forces acting at surface-modified AFM probes/various substrates in air and water

\begin{tabular}{ccccc}
\hline \multirow{2}{*}{$\begin{array}{c}\text { Analytical } \\
\text { condition }\end{array}$} & Sample & \multicolumn{3}{c}{ Force (nN) } \\
\cline { 3 - 5 } & & $\mathrm{Au}$ & $\mathrm{CH}_{3}$ & $\mathrm{COOH}$ \\
\hline \multirow{2}{*}{ Air } & Cellulose & $31 \pm 3$ & $21 \pm 1$ & $22 \pm 2$ \\
& Oxidized Al & $22 \pm 1$ & $25 \pm 2$ & $57 \pm 5$ \\
\multirow{2}{*}{ Water } & Cellulose & $0.66 \pm 0.16$ & $1.29 \pm 0.13$ & $1.26 \pm 0.10$ \\
& Oxidized Al & $0.79 \pm 0.16$ & $1.77 \pm 0.13$ & $5.02 \pm 0.13$ \\
\hline
\end{tabular}

in oil were found to be effectively lubricated by the presence of fatty acid molecules whose polar groups were attached to the aluminum oxide layers [30]. Formation of self-assembled monolayers of fatty acids on aluminum oxide surface was also reported, and such monolayers could be partially removed by rinsing with $\mathrm{CHCl}_{3}[31,32]$. These findings may be associated with a specific attraction between carboxyl groups of model size components and oxidized aluminum substrate, and are presumably closely related to the macroscopic sizing appearance demonstrated in Figs. 1 and 2. In addition, it has been reported that aluminum compounds are homogeneously scattered in paper matrix [16]. The implication is that the size components were also well scattered in the paper matrix through an attractive interaction, resulting in desirable sizing effects.

\subsection{Sizing application to porous inorganic fiber- network material (glass filter)}

That a unique interaction between fatty acids and oxidized aluminum components contributes to good sizing appearance was demonstrated using AFM analysis

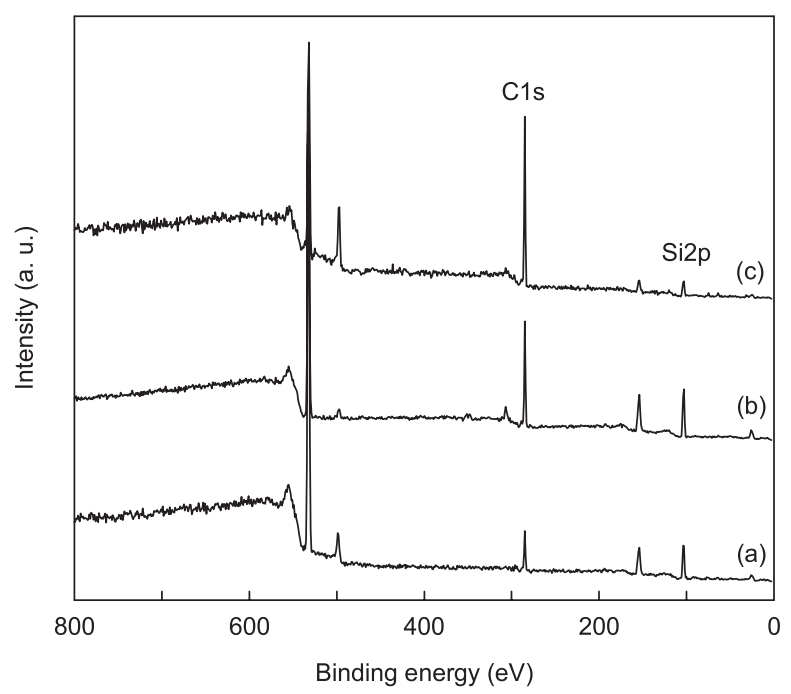

Fig. 5 XPS survey spectra of glass filters (a) without, and $(\mathrm{b}, \mathrm{c})$ with sizing treatment using $0.5 \%(\mathrm{w} /$ v) $\mathrm{SPA} / \mathrm{CHCl}_{3}$ solution. The sized glass filters were pretreated with (b) $0 \%$, and (c) $0.2 \%$ alum solution. in the previous section. Subsequently, the specific interaction was utilized to impart water-repellency to other fiber-network materials, in particular a glass filter. Commercial glass filter mats were sequentially treated with an aqueous alum solution and a nonaqueous SPA/ $\mathrm{CHCl}_{3}$ solution. The sized glass filters treated at various alum concentrations were analyzed via XPS and CA measurements. Regardless of pretreatment with alum, strong C1s peaks were detected in the XPS survey spectra (Fig. 5), indicating large amounts of size components on the glass filters after soaking in $0.5 \%(\mathrm{w} / \mathrm{v}) \mathrm{SPA} / \mathrm{CHCl}_{3}$ solution.

The atomic ratio of $\mathrm{C} 1 \mathrm{~s}$ derived from fatty acids to $\mathrm{Si} 2 \mathrm{p}$ from glass fibers was measured to evaluate the surface size abundance on the glass filter matrix. As shown in Fig. 6, the atomic ratio $(\mathrm{C} / \mathrm{Si})$ increased linearly with an increase in alum concentration in the alum pretreatment procedure. Moreover, increasing alum concentration brought about higher CA values $\left(\approx 120^{\circ}\right)$ of water droplets on the sized glass filter surfaces, thus enhanced hydrophobicity, while the contact angle was $c a$. $0^{\circ}$ without alum pretreatment. These results strongly suggested that addition of hydrophobic fatty acids alone could not impart water repellency to porous glass filters. Hence, a clear size appearance in the presence of aluminum components was presumably induced by homogeneously anchoring the size components to the glass filter surface. The origin of the effect is the attractive force acting at the interface of free carboxyl groups of fatty acids and oxidized aluminum components.

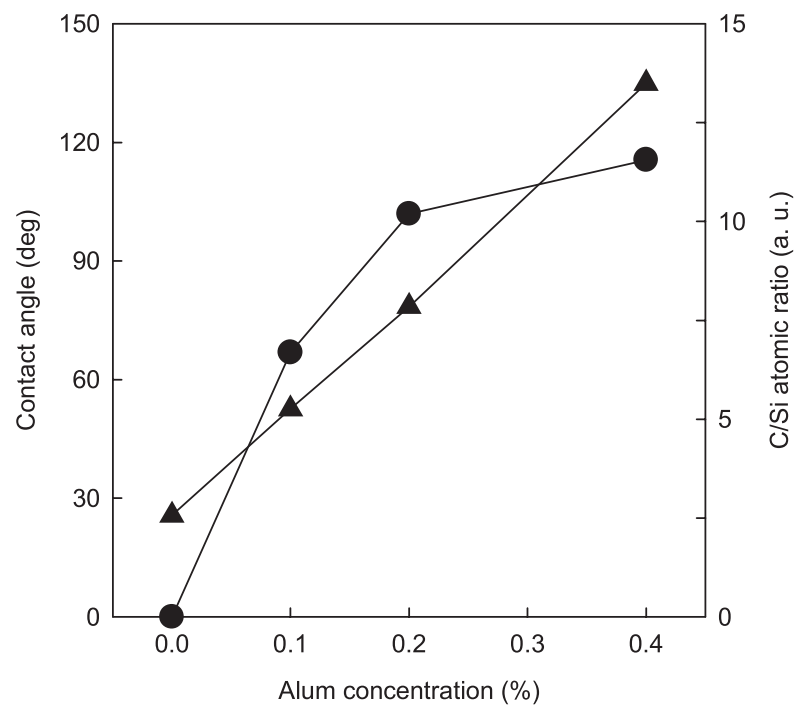

Fig. 6 Water $\mathrm{CA}$ values (circles) and $\mathrm{C} / \mathrm{Si}$ atomic ratio (triangles) on the surface of glass filters sized with $0.5 \%(\mathrm{w} / \mathrm{v}) \mathrm{SPA} / \mathrm{CHCl}_{3}$ solution. The glass filters were pre-soaked in $0-0.4 \%$ alum solution, followed by adding sodium hydroxide solution. 
Such an attractive force must be an essential factor for the sizing appearance of porous fiber-network architectures, and is expected to have potential applications for design of paper-like materials.

\section{Conclusion}

Specific interaction between a carboxyl terminus of fatty acid and oxidized aluminum is indicated by AFM force-distance analysis using surface-modified probes with either methyl or carboxyl moiety as an end group. Significant attractive forces are detected at the carboxylterminated AFM probe/oxidized aluminum interface in air and water, respectively. The combination of fatty acid and oxidized aluminum provides effective water-repellency to not only organic pulp sheet but also inorganic glass filter. It is suggested that the attractive interaction between the carboxyl group in acid sizes and oxidized aluminum components in the hydrophilic matrix must play an essential role in the wetting characteristics of porous fiber-network architectures.

\section{Acknowledgments}

This research was supported by a Research Fellowship for Young Scientists from the Japan Society for the Promotion of Science (S.Y.), and by a Grant-inAid for Young Scientists (S: 21678002) from the Ministry of Education, Culture, Sports, Science and Technology of Japan (T.K.).

\section{References}

1. S. Fukahori, H. Ichiura, T. Kitaoka, and H. Tanaka, Appl. Catal. B: Environ., 46, 453 (2003).

2. J.-G. Huang, I. Ichinose, and T. Kunitake, Angew. Chem. Int. Ed., 45, 2883 (2006).

3. S. Egusa, S. Yokota, K. Tanaka, K. Esaki, Y. Okutani, Y. Ogawa, T. Kitaoka, M. Goto, and H. Wariishi, J. Mater. Chem., 19, 1836 (2009).

4. J. M. Gess, "Paper Chemistry, second edition", (J. C. Roberts Ed.), Chapman \& Hall, New York, p. 120 (1996).

5. E. J. Vandenberg and H. M. Spurlin, Tappi J., 50, 209 (1967).

6. R. W. Davison, J. Pulp Pap. Sci., 14, J151 (1988).

7. T. Kitaoka, A. Isogai, and F. Onabe, Nord. Pulp Pap. Res. J., 12, 26 (1997).
8. T. Kitaoka, A. Isogai, F. Onabe, and N. Suguri, Nord. Pulp Pap. Res. J., 12, 182 (1997).

9. T. Kitaoka, A. Isogai, F. Onabe, and T. Endo, Nord. Pulp Pap. Res. J., 16, 96 (2001).

10. Y. Ishida, H. Ohtani, T. Kano, S. Tsuge, and T. Yano, Tappi J., 77, 177 (1994).

11. T. Kitaoka, A. Isogai, and F. Onabe, Nord. Pulp Pap. Res. J., 10, 253 (1995).

12. K. Ito, A. Isogai, and F. Onabe, J. Pulp Pap. Sci., 25, 222 (1999).

13. F. Wang and H. Tanaka, J. Appl. Polym. Sci., 78, 1805 (2000).

14. T. Kitaoka, A. Isogai, and F. Onabe, Nord. Pulp Pap. Res. J., 15, 177 (2000).

15. F. Wang, H. Tanaka, T. Kitaoka, and M. A. Hubbe, Nord. Pulp Pap. Res. J., 15, 416 (2000).

16. M. Kato, A. Isogai, and F. Onabe, J. Wood Sci., 46, 75 (2000).

17. T. Kitaoka, H. Yamamoto, R. Nishino, and $H$. Tanaka, Sen'i Gakkaishi, 57, 212 (2001).

18. H. Yamamoto, T. Kitaoka, and H. Tanaka, Sen' $i$ Gakkaishi, 59, 266 (2003).

19. H. Yamamoto, T. Kitaoka, and H. Tanaka, J. Pulp Pap. Sci., 30, 136 (2004).

20. T. R. Arnson and R. A. Stratton, Tappi J., 66, 72 (1983).

21. M. Kato, A. Isogai, and F. Onabe, J. Wood Sci., 44, 361 (1998).

22. J. S. Ellis, S. Allen, Y. T. A. Chim, C. J. Roberts, S. J. B. Tendler, and M. C. Davies, Adv. Polym. Sci., 193, 123 (2006).

23. TAPPI Test Methods, T227 om-94 (1995).

24. TAPPI Test Methods, T200 om-89 (1995).

25. TAPPI Test Methods, T 205 om-88 (1995).

26. S. Yokota, T. Kitaoka, and H. Wariishi, Appl. Surf. Sci., 253, 4208 (2007).

27. C. D. Frisbie, L. F. Rozsnyai, A. Noy, M. S. Wrighton, and C. M. Lieber, Science, 265, 2071 (1994).

28. JIS Methods, P 8122 (2004).

29. K. Matsuyama, S. Yokota, T. Kitaoka, and H. Wariishi, Sen'i Gakkaishi, 62, 89 (2006).

30. R. S. Timsit, and C. V. Pelow, J. Tribol., 114, 150 (1992).

31. Y.-T. Tao, J. Am. Chem. Soc., 115, 4350 (1993).

32. K. Oberg, P. Persson, A. Shchukarev, and B. Eliasson, Thin Solid Films, 397, 102 (2001). 